Journal of Fluid Mechanics

http://journals.cambridge.org/FLM

Journal of

Additional services for Journal of Fluid Mechanics:

Fluid Mechanics

Email alerts: $\underline{\text { Click here }}$

Subscriptions: $\underline{\text { Click here }}$

Commercial reprints: $\underline{\text { Click here }}$

Terms of use : $\underline{\text { Click here }}$

\title{
Dust resuspension by the flow around an impacting sphere
}

\section{EAMES and S. B. DALZIEL}

Journal of Fluid Mechanics / Volume 403 / January 2000, pp 305 - 328

DOI: 10.1017/S0022112099007120, Published online: 08 September 2000

Link to this article: http://journals.cambridge.org/abstract_S0022112099007120

How to cite this article:

I. EAMES and S. B. DALZIEL (2000). Dust resuspension by the flow around an impacting sphere. Journal of Fluid Mechanics, 403, pp 305-328 doi:10.1017/S0022112099007120

Request Permissions : $\underline{\text { Click here }}$ 


\title{
Dust resuspension by the flow around an impacting sphere
}

\author{
By I. EAMES† AND S. B. DALZIEL \\ Department of Applied Mathematics and Theoretical Physics, University of Cambridge, \\ Silver Street, Cambridge CB3 9EW, UK
}

(Received 20 February 1999 and in revised form 11 August 1999)

\begin{abstract}
A rigid body colliding with a layer of dust is capable of resuspending dust through two distinct mechanisms: a ballistic mechanism, where kinetic energy is transferred from the impacting body to dust particles through direct contact, and a hydrodynamic mechanism, where dust particles are resuspended by the flow disturbance generated by the body. In this paper, we study the hydrodynamic resuspension mechanism by considering the flow around a sphere moving either towards or away from a wall. Experiments were performed using a sphere translating at a constant velocity for Reynolds number, $R e$, in the range 300 to 3500 , and at varying angles of approach and departure from a wall. A wider range of $R e$ was investigated by releasing dense rigid spheres above the wall. The high Reynolds number flow past a steadily translating sphere is characterized by a recirculating wake region behind the sphere. When the sphere approaches the wall and stops on making contact with it, the wake vortex which is initially behind the sphere threads over the sphere's surface, generating a secondary vortex ring. The coherent structure, composed of the wake and secondary vortices, strikes the wall and pushes fluid or dust, initially adjacent to the wall, to one side. The resuspension of dust particles of diameter $b$ which are initally at rest on the wall is governed by a particle Shields' parameter, $\theta_{p}$, based on the sphere's impact velocity, $U: \theta_{p}=\rho_{f} U^{2} /\left(\rho_{p}-\rho_{f}\right) b g$, where $\rho_{p}$ and $\rho_{f}$ are respectively the density of the dust particles and fluid. The resuspension criterion is a function of particle Reynolds number, $R e_{p}$, based on the diameter and fall velocity of the dust particles and occurs when $\theta_{p} \geqslant \theta_{p, c}$ where $\theta_{p, c} \approx 3.0$ for $R e_{p} \gtrsim 1$, and $\theta_{p, c} \approx 5.0 / R e_{p}^{1 / 2}$ for $R e_{p} \lesssim 1$. The geometry of the region of dust resuspended by the sphere was studied as a function of the impact velocity, angle of impact and the properties of the dust particles. When the sphere impacts a thick layer of dust, the volume concentration of resuspended dust is sufficiently high to generate a particle-driven gravity current which transports the dust far from the point of impact. The dynamics of the gravity current were determined as a function of dust particle properties and size of the impacting sphere.

A sphere moving impulsively from rest away from a wall is found not to play a significant role in the resuspension of dust; however trailing vorticity generated on the surface of the sphere advects a large volume of fluid away from the wall, which may contain dust already in suspension.
\end{abstract}

$\dagger$ Present address: Department of Mechanical Engineering, University College London, Torrington Place, London WC1E 7JE, UK. 


\section{Introduction}

Fine airborne particulate such as dust occurs in great abundance in soils, sediments and stockpiled materials such as mineral ores or agricultural grain. Such quantities pose an important environmental problem, particularly because dust has the propensity to travel great distances in suspension and can also directly enter the bronchial tract of mammals, causing severe respiratory problems. Dust loss from soils is also responsible for the reduction of soil fertility because the largest component of soil fertility is contained in the smallest particle size fraction. Central to assessing the environmental risk posed by stockpiled sediments is an accurate estimate of the vertical mass flux of dust removed from the sediment surface. A large number of field and laboratory studies have been performed to determine how the vertical mass flux of dust varies with wind speed and soil properties (Gillette et al. 1974). Whilst progress has been made in characterizing the vertical mass dust flux released from the ground in terms of soil type and moisture, relatively little is known about the processes responsible for dust particles leaving the ground in the first place.

A great deal of research has been directed towards understanding the transport of unimodal sediments by the wind (Bagnold 1941). The wind provides the mechanism of sediment removal from the ground through the action of aerodynamic lift and transport, in the wind or along the ground, by the action of a drag force. The strength of the wind is characterized in terms of a friction velocity, $u_{*}$, which is often related to the wind velocity in the boundary layer by a logarithmic profile $U(z)=\left(u_{*} / \kappa\right) \log \left(z / z_{0}\right)$, where $\kappa=0.4$ is von Kármán's constant and $z_{0}$ the roughness height. When the friction velocity is low, the sediment is undisturbed, but as the friction velocity increases, a critical value, $u_{*, c}$, is reached when sediment motion is initiated. At this point the aerodynamic lift force on individual particles is sufficient to overcome the buoyancy or cohesive force (Absil \& Beugeling 1984) which keeps them anchored to the ground. The critical condition for the removal of sediment particles of diameter $a$ from the ground is usually represented in terms of a Shields' parameter, $\theta$, defined as $\theta=\rho_{f} u_{*}^{2} /\left(\rho_{p}-\rho_{f}\right) g a$, which may be interpreted as the ratio of the inertial lift force based on the critical friction velocity, $O\left(\rho_{f} u_{*}^{2} a^{2}\right)$, and the buoyancy force $O\left(\left(\rho_{p}-\rho_{f}\right) g a^{3}\right)$, where $\rho_{p}, \rho_{f}$ are respectively the density of the particles and fluid. In general, small particles are difficult to remove from the ground by the wind because they lie within the viscous sublayer and so the aerodynamic lift force acting on them is weak, and large particles are difficult to remove because they are heavy. Soil particles (density $\rho_{p}=2500 \mathrm{~kg} \mathrm{~m}^{-3}$ ) most susceptible to dislodgement by the wind have a typical diameter of $100 \mu \mathrm{m}$, corresponding to a minimum critical friction velocity of $u_{*, c}=0.15 \mathrm{~m} \mathrm{~s}^{-1}$. The mode of particle transport by the wind, following sediment removal from the ground, depends on the ratio of the friction velocity, $u_{*}$, which is a measure of the turbulent velocity fluctuations in the wind, to the terminal fall speed of the particle, $v_{T}$. The finest constituent of soil, which we hereafter refer to as 'dust', has a typical diameter less than $50 \mu \mathrm{m}$ and travels by suspension in the wind when $v_{T} \lesssim u_{*}$. 'Sand' particles of diameter $150-600 \mu \mathrm{m}$ travel with parabolic hops along the ground - this mode of transport is called saltation and occurs when $v_{T} \gtrsim u_{*}$. Particles with diameters greater than $600 \mu \mathrm{m}$ are too large to enter the wind, except under extreme conditions, and instead roll or 'creep' along the ground.

Bagnold (1941) demonstrated experimentally that although a thin layer of dust is difficult to resuspend from the ground because it lies within the viscous sublayer, it is readily liberated when a small quantity of sand is introduced upstream of the layer of dust. As the sand particles saltate across the layer of dust, they disturb the dust particles leading to dust release from the surface. We categorize the disturbance caused 
by a particle colliding with a layer of dust as either ballistic, where saltating particles collide with dust particles, break the cohesive inter-particle dust bonds and eject fine particulate from the bed, or hydrodynamic, where the flow around saltating particles colliding with or leaving the ground is responsible for resuspending particles. The aim of this paper is to examine in detail the hydrodynamic resuspension mechanism.

The ballistic mechanism describes when a particle colliding with a sediment breaks the cohesive bonds which exist between particles, providing the kinetic energy of the impacting particle is sufficiently large, and ballistically ejecting particles both larger and smaller in size. Rice, Willetts \& McEwan (1996) examined the microscopic process of dust ejection by sand particles $(250-500 \mu \mathrm{m}$ in diameter) colliding with an unaggregated bed of fine particles whose diameters were less than $53 \mu \mathrm{m}$. The collisions were recorded on high-speed film and subsequent examination of the surface texture of the sediment by a scanning laser showed elliptical craters, where dust was removed. The mass of dust removed by the collision of a single saltating grain $S_{a}$ could be accurately measured, and was found to be proportional to the kinetic energy lost to the sediment by the saltating particle colliding with the ground, in agreement with abrasion studies of Greeley \& Iverson (1985). Shao, Raupach \& Findlater (1993) studied the macroscopic process of dust release by measuring the horizontal mass flux of dust generated by sand particles saltating across a layer of dust. They proposed that the horizontal mass flux of dust, $q_{d}$, is proportional to the collision rate per unit area, $n$, and the mass of dust ejected during each collision of a saltating particle with the dust layer, $S_{a}: q_{d}=n S_{a}$. The predicted variation of $q_{d}$ with friction velocity was found to be consistent with experimental measurements; we also note that this variation is consistent with the results of Rice et al. (1996). In the studies described above, the mass of dust released by a saltating particle colliding with a rich bed of dust was quantified; however the processes which mediate the transport of suspended dust away from the ground were not examined. In addition, the mechanisms or processes through which saltating particles resuspend thick dust deposits are not necessarily dominant when the dust is scattered across a sediment surface.

The hydrodynamic mechanism describes when the flow around a body moving near a layer of dust is responsible for dislodging and resuspending dust particles. Owen (1980) argued that the range of influence of an effective mechanism of dust resuspension must be larger than the contact area between a saltating particle and the ground because the probability that saltating particles strike and dislodge dust particles through this mechanism is too low to account for the observed rates of dust release. Following this, Owen (1980) proposed the flow around the sand particle leaving the ground as a candidate for the principal mechanism of dust resuspension. Owen (1980) originally examined the dynamics of dust particles in the flow around a body moving perpendicularly to a wall. More recently, Owen (1986) performed an experiment to illustrate the effect of a sphere colliding with a wall in resuspending dust. Owen observed the intense wake vortex of the sphere threading over the surface of the sphere, subsequently striking the wall, removing and resuspending the dust, and concluded that the impact of the sphere with the wall was the dominant mode of resuspension.

The main purpose of this paper is an examination of hydrodynamic resuspension by a sphere moving near a wall. The study presented here has three new components: first, the high-Re flow around a body moving towards or away from a wall is examined. Whilst the flow around a body moving near a wall has been examined for low Re (Brenner 1961), the corresponding case of high $R e$ has been restricted to a discussion of space probes landing on Titan (Lorenz 1994). An examination of the high-Re flow past a rigid body moving near a wall has a bearing on other multiphase 


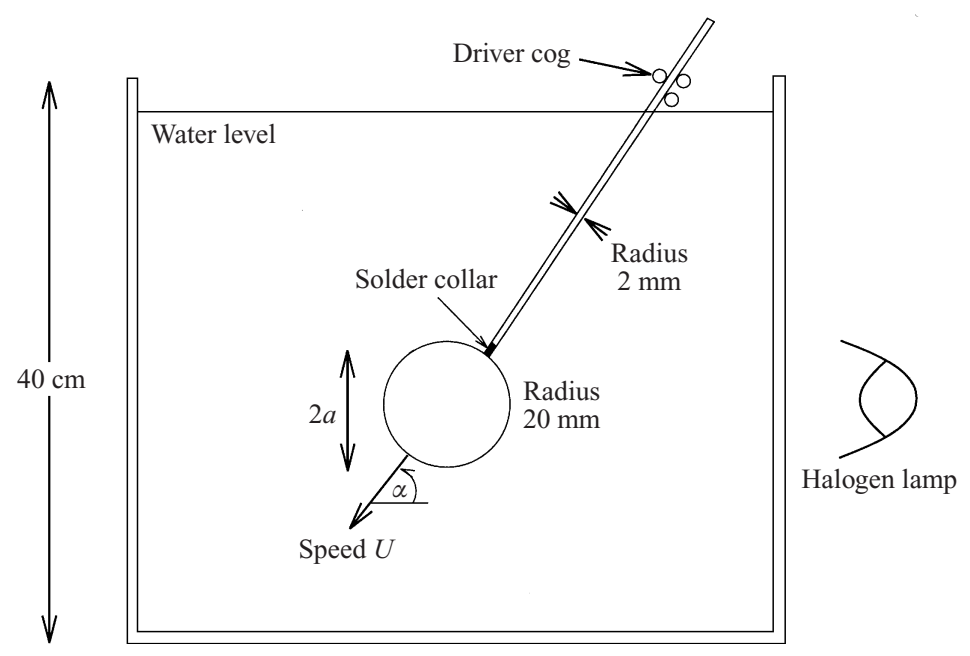

FIGURE 1. Schematic illustration of the experimental apparatus used to study the flow around a sphere moving towards or away from a rigid wall.

problems, such as the flow generated by 'dirty' bubbles rising to a free surface, which play an important role in interfacial gas transfer in the ocean. As we shall see, the resuspension process is dominated by the wake vortex colliding with the wall. The flow generated by a ring vortex striking a wall has been studied in detail (see Walker et al. 1985 and references therein), and recently the resuspension of dust by vortices impinging on a wall has been studied experimentally (Wells 1992). However, the role played by the flow around a body in resuspending dust has not been studied in detail, and this remains the primary focus of this paper. Secondly, the critical condition for this layer of particles to be disturbed and resuspended by the flow generated by a sphere colliding with the ground is determined. The resuspension criterion has not hitherto been determined and is relevant to a wide range of problems such as the resuspension by containers dumped into the sea which impact the sea bed. Thirdly, the dust particle dynamics following resuspension is examined for the case when the concentration of resuspended material is high and a particle-driven gravity current is generated. The resuspension process controls the initial mass of dust resuspended, and the subsequent dynamics of the gravity current is characterized experimentally.

This paper is structured as follows: in $\S 2$, the flow around a sphere moving towards a wall is discussed. The role played by this flow in resuspending dust particles is studied experimentally in $\S 3$, where we treat the case of the sphere impacting a thin and thick layer separately. The flow around a sphere moving impulsively from rest away from a wall is presented in $\S 4$. Finally our conclusions are drawn and put into the broader context in $\S 5$.

\section{Flow around a sphere moving towards a wall}

The flow around a polished Perspex sphere (radius $a=20 \mathrm{~mm}$ ) moving near a wall was studied in a $40 \mathrm{~cm}$ cubed Perspex tank which was filled with tap water (figure 1). The motion of the sphere was controlled by mounting it on the end of a $2 \mathrm{~mm}$ radius rigid rod which was driven by a simple traverse consisting of two ' $\mathrm{v}$ ' idler wheels on one side and a spring-loaded rubber driving wheel on the other. Two additional guides were employed to maintain alignment and a guide rail to prevent rotation. 
The Perspex sphere was impulsively started from rest at the top of the tank and traversed with a constant velocity $U$ towards the wall. As the sphere descends the wake vortex grows and the flow becomes well-established. This flow is characterized by the Reynolds number, $R e=2 U a / v$, where $a$ is the sphere's radius and $v$ the fluid kinematic viscosity. This flow has been studied in detail by a number of researchers and the main results are summarized by Clift, Grace \& Weber (1978): when $R e<130$, the flow past a sphere moving in an unbounded domain is steady but the wake becomes unsteady for $130<R e<400$. At higher Reynolds number $(R e>400)$, the wake is unstable and liable to shed vortices, and the precise form of the shed vortices was studied by Magarvey \& Maclarehy (1968). The vortex shedding frequency of the wake is characterized by the Strouhal number, $S r=2 a f / U$, which is defined as the ratio of advective timescale of translation to shedding timescale $(1 / f)$. Consequently, a vortex is expected to shed from the wake as the sphere moves through a distance $2 a / S t$.

We examined the flow for Reynolds numbers in the range $R e=300-3500$, and impact angles $\alpha=64^{\circ}-90^{\circ}$ relative to the wall. The Strouhal number increases from 0.1 to 0.2 as $R e$ increases from 300 to 3500 , so we expect the wake to shed a vortex as the sphere traverses a distance of $20 a$ to $10 a$. Since the aim was to examine the role played by the wake vortex behind the sphere in resuspending dust, we impulsively started the sphere a vertical distance $15 a$ from the wall. This distance was chosen as a compromise between having a well-established flow prior to the sphere impacting the wall, and requiring that a vortex is not shed before the sphere strikes the wall.

\subsection{Wake structure and vortex dynamics}

Figure 2 shows the evolution of the dyed wake behind the sphere, following its impact with the wall, and this sequence is typical of those observed at high Re. Here, the flow is characterized by $R e=850, \alpha=90^{\circ}$, and the time difference between each image, $2 a / U$, corresponds to the time taken for the sphere to translate one diameter. Figure 2 (b) corresponds to the time when the sphere first makes contact with the wall.

The outline of the primary wake prior to the sphere impacting the wall is seen in figure $2(a, b)$. Following the impact, the wake continues to move towards the wall because of its inertia and the self-induced motion associated with its vorticity field. As the wake vortex passes over the sphere, the vortical elements are stretched so that the vorticity increases, whilst the circulation associated with the vortical elements remains conserved. Vorticity of the opposite sign to the wake vortex is generated on the sphere surface as it overtakes the sphere. Both the curvature of the surface of the sphere and growing boundary layer contribute to an adverse pressure gradient along the surface of the sphere which decelerates the flow, leading to boundary layer separation and the production of a secondary vortex of the opposite sign to that of the wake vortex (clearly seen in figure $2 c, d$ ). In figure $2(d)$, we see the coherent structure composed of the wake and secondary vortex leave the surface of the sphere at an angle of approximately $135^{\circ}$ to the rod and strike the wall. The secondary vortex is subsequently stretched by the wake vortex and becomes sheet-like, as seen in figure $2(f)$ where the secondary vortex is of approximate thickness $0.2 a$ and extends radially between $1.0 a$ and $2.2 a$ from the centreline. In figure $2(e)$, the secondary vortex breaks into two parts: one component is advected around the wake vortex, and the second component remains trapped close to the point of contact between the sphere and wall.

In figure $2(g-j)$, the flow induced by the secondary vortex causes the wake vortex to be deflected away from the wall and the combined structure rotates. The coherent structure strikes the wall and continuously generates vorticity of the opposite sign to the wake vortex on the wall. At later times, the primary or wake vortex is 

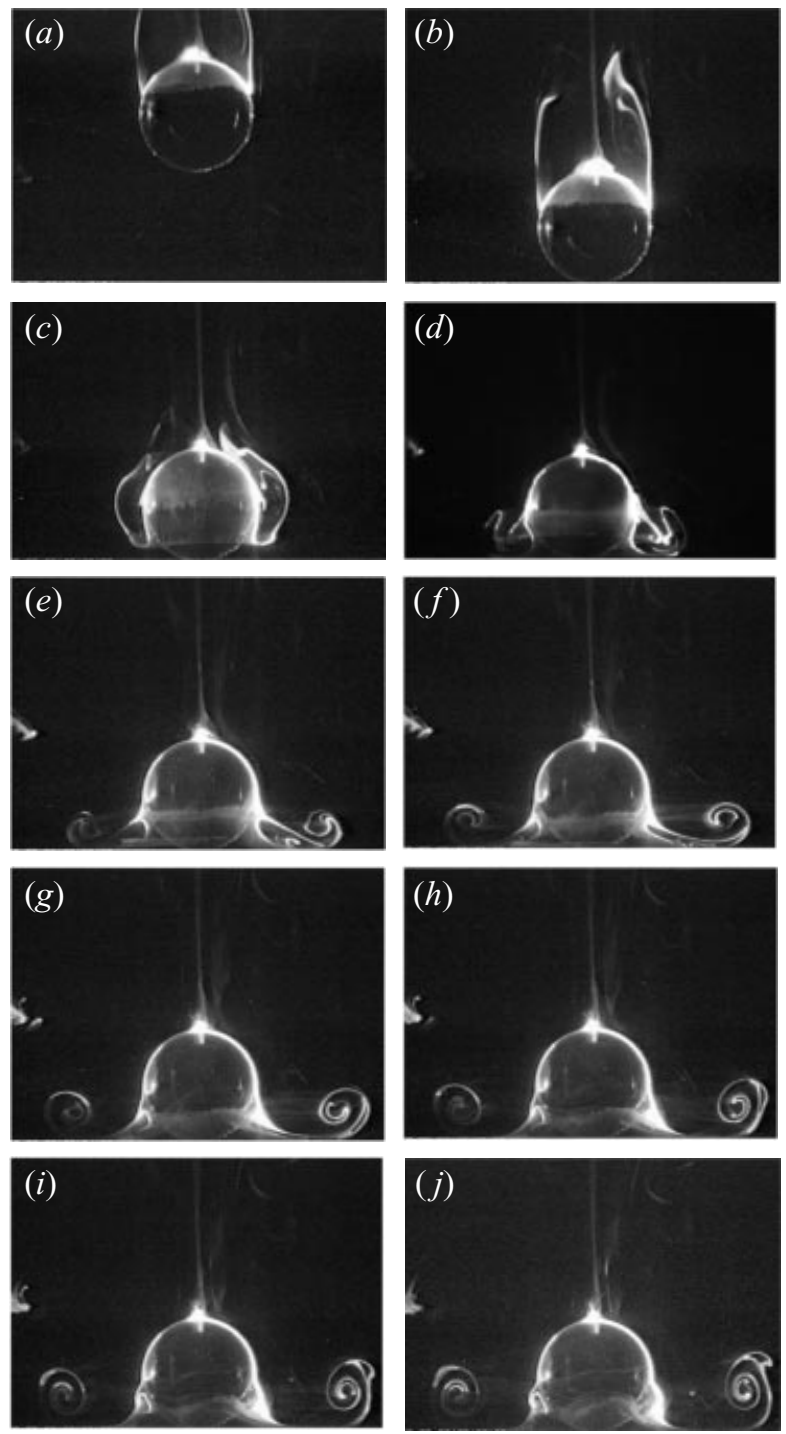

FIGURE 2. The sphere approaches the wall, and impulsively stops on making contact with the wall. The flow is characterized by $\operatorname{Re}=850, \alpha=90^{\circ}$, and the wake is dyed by electrolytic precipitation of tin chloride. The sequence of images illustrates the dynamics of the wake vortex following the impact of the sphere, and the time difference between adjacent figures is $2 a / U$.

enveloped by vorticity of the opposite sign and shielded from the effect of the wall. Additional experiments indicated that even when the initial flow is three-dimensional and turbulent, the final state appears to be one in which the wake vortex stops propagating a finite distance from the centreline, and the strength of the wake vortex subsequently decays due to viscous dissipation.

The flow adjacent to the wall was examined by introducing a thin layer of fluorescein dye adjacent to the wall. Figure $3(a-f)$ shows the motion of a thin dye layer due to the impact of the sphere at $R e=850$. The initial response is for the dye to be pushed to one side by the impact of the wake and secondary vortex. Subsequently, the secondary vortex is wrapped around the primary vortex, compressed (as seen in 

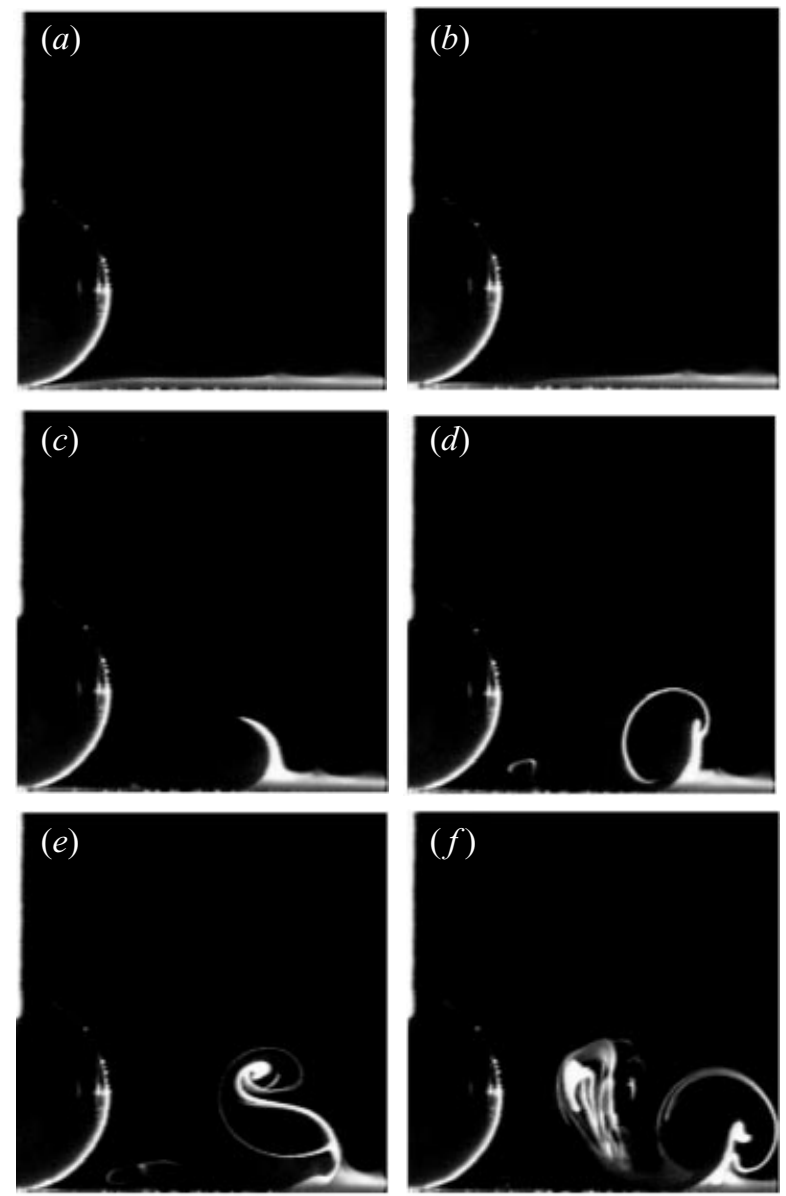

FIGURE 3. When the sphere collides with the wall, the wake and secondary vortex strike the wall pushing the thin layer of fluorescein dye to one side, which is subsequently entrained by the wake vortex. Here, the flow is characterized by $R e=850, \alpha=90^{\circ}$. The first image, $(a)$, corresponds to the time when the sphere makes contact with the wall and the time difference between the subsequent images is $2 a / U$.

figure $3 e$ ), and becomes unstable (as seen in figure $3 f$ ). The cause of this azimuthal instability is the straining of the secondary vortex by the wake vortex; this mechanism has been studied in detail for vortex rings impinging walls (Orlandi \& Verzicco 1993; Swearingen, Crouch \& Handler 1995).

When the sphere strikes the wall at an oblique angle, the flow is not axisymmetric about the collision point because the wake vortex ring is stretched by varying degrees around its perimeter. The vortex dynamics are initially unaffected by the presence of the wall. As before, the wake vortex generates a secondary vortex and the combined coherent structure detaches at an angle of $135^{\circ}$ to the rod. The subsequent dynamics of the wake vortex were observed to change as $R e$ increased. From the dyed wake and dyed layer experiments, we observed that when the sphere struck the wall at an oblique angle $\left(\alpha=72^{\circ}\right)$, the wake vortex downstream of the point of contact did not strike the wall when $R e \lesssim O(1000)$, and consequently, the fluid adjacent to the wall was not disturbed. When $R e \gtrsim O(1000)$, the wake vortex was observed to strike the wall. Figure $4(a-f)$ shows the flow generated by a sphere impacting at an 

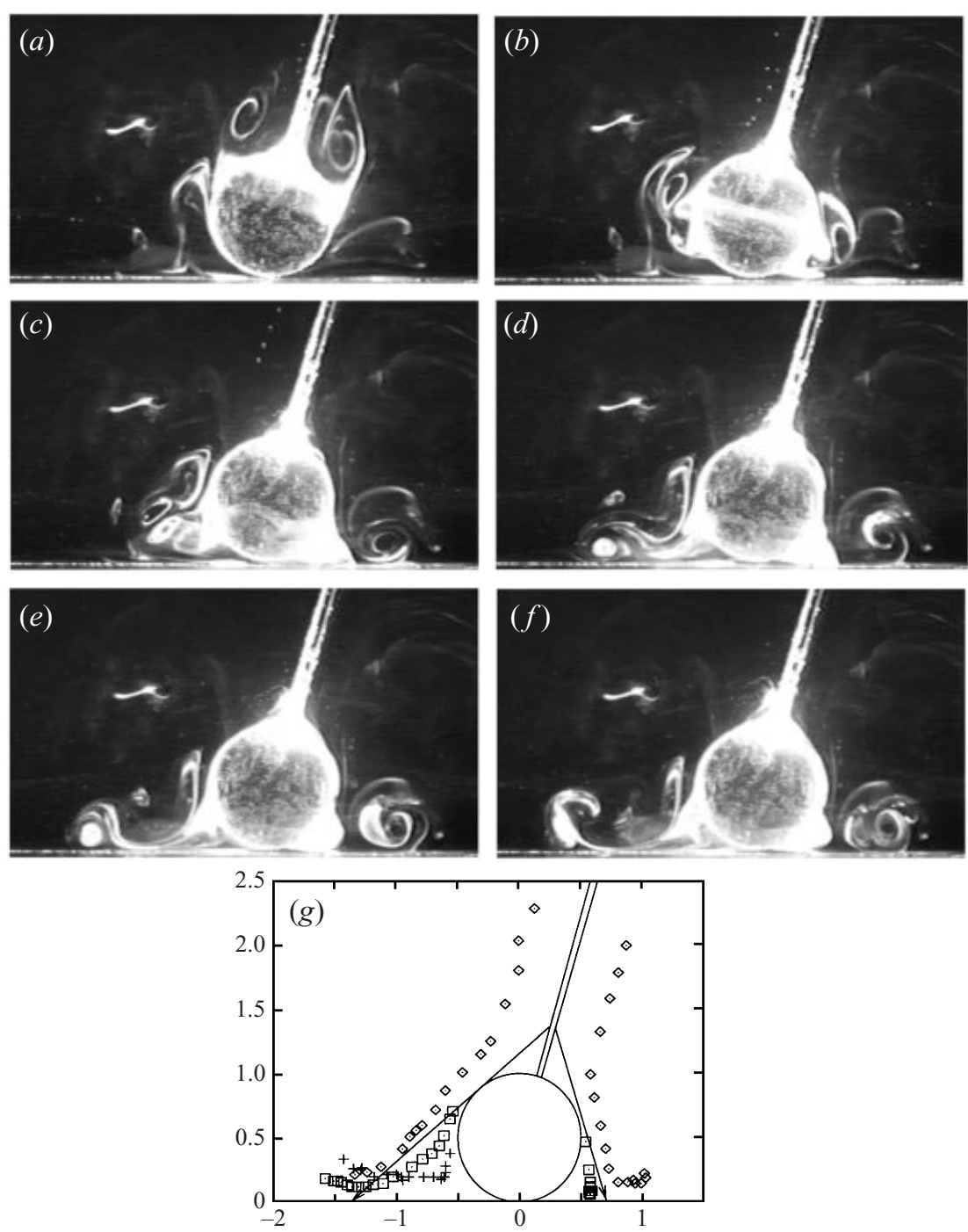

FIGURE 4. Series of images showing the collision of the sphere at an oblique angle $\left(\alpha=72^{\circ}\right)$ with the wall. The flow is characterized by $R e=1470$. The wake is visualized by precipitating tin chloride at the neck of the rod. Image $(a)$ corresponds to the time of contact between the wall and sphere. The time difference between subsequent images is $1.0 a / U$. $(g)$ The positions of the primary $(\diamond)$, secondary $(\square)$ and separated primary vortices $(+)$ are indicated (the time difference between the centres of the vortices is $a / 4 U$ ).

oblique angle $\alpha=72^{\circ}, R e=1470$; the time difference between each image is $a / U$. Figure $4(\mathrm{~g})$ shows the positions of the wake vortex, secondary vortex, and part of the wake vortex which has been separated from the main wake. Although the flow is three-dimensional, we shall discuss the flow on the left-hand and right-hand side of the sphere separately.

On the right of the sphere, the coherent structure composed of the wake and secondary vortex travels a shorter distance prior to colliding with the wall at a steep angle $135^{\circ}-\alpha=63^{\circ}$ (compared to $45^{\circ}$ when the sphere impacts the wall normally). As indicated in figure 4, the coherent structure is lifted up from the wall and rotates, prior to impacting the wall again. Similar observations were made by Walker et al. 

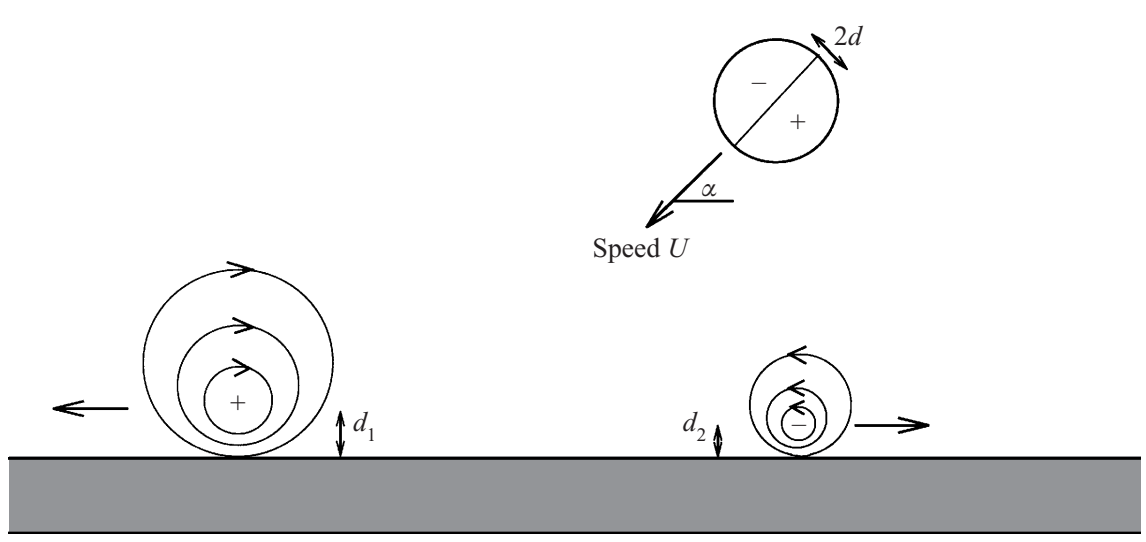

FIGURE 5. Schematic of a two-dimensional dipolar vortex impacting on a wall. This example is used to gain some insight into the case when the sphere, and consequently the wake vortex, approaches the wall at an oblique angle.

(1987) regarding the dynamics of secondary and primary vortices following the impact of a vortex ring on a wall.

On the left of the sphere, the primary and secondary vortices travel at a shallower angle to the wall $\left(\alpha-45^{\circ}=27^{\circ}\right)$, propagating further, prior to colliding with the wall. The coherent structure, composed of the wake and the secondary vortex, turns and as it continues to propagate is deflected away from the wall. The deflection of the wake vortex away from the wall is affected by the strength of the secondary vortex: as the strength of the secondary vortex decreases, the deflection of the wake vortex away from the wall becomes more pronounced. The strength of the secondary vortex appears to increase as $R e$ increases: when $R e \leqslant O(1000)$, the secondary vortex is weak and the deflection of the wake vortex away from the wall is pronounced, and for $R e \geqslant O(1000)$ the wake vortex strikes the wall.

The flow generated by a sphere impacting a wall obliquely has an important bearing on the resuspension process. To facilitate our interpretation of figure 4 we examined the dynamics of a dipolar vortex striking a wall obliquely with an angle $\alpha$ (figure 5). The vortex dipole initially consists of line vortices of circulation $\pm \kappa$ and separation $d$, and therefore propagates with a velocity $U=\kappa / 2 \pi d$. Following the collision of the vortex dipole with the wall, the vortices separate and ultimately propagate parallel to the wall, but in opposite directions (figure 5). We may understand this behaviour by considering the impulse $U d^{2}$ associated with the translation of a vortex. The concept of fluid impulse is analogous to linear momentum and the component of impulse parallel to the wall is conserved during the collision. As a result of this, the difference between the final separation of the line vortices and the wall must satisfy $d_{1}-d_{2}=2 d \cos \alpha$. Energy is conserved during the collision ensuring that $d_{1} d_{2}=d^{2}$ (Batchelor 1967). Therefore the separations between the line vortices and the wall ultimately tend to $d_{1} / d=\cos \alpha+\sqrt{\cos ^{2} \alpha+1}$, and $d_{2} / d=\sqrt{\cos ^{2} \alpha+1}-\cos \alpha$. These results indicate that a two-dimensional vortex striking a wall obliquely generates the line vortex propagating to the left in figure 5 , which is at a distance $d_{1}>d$ from the wall and travels more slowly than the initial vortex dipole. In contrast the line vortex on the right-hand side of figure 5, propagates with a velocity larger than $U$.

Although the above model of the flow is two-dimensional and inviscid, it demonstrates the immediate consequence of the component of fluid impulse parallel to the wall and energy being conserved during the collision. Two-dimensional dynamics 


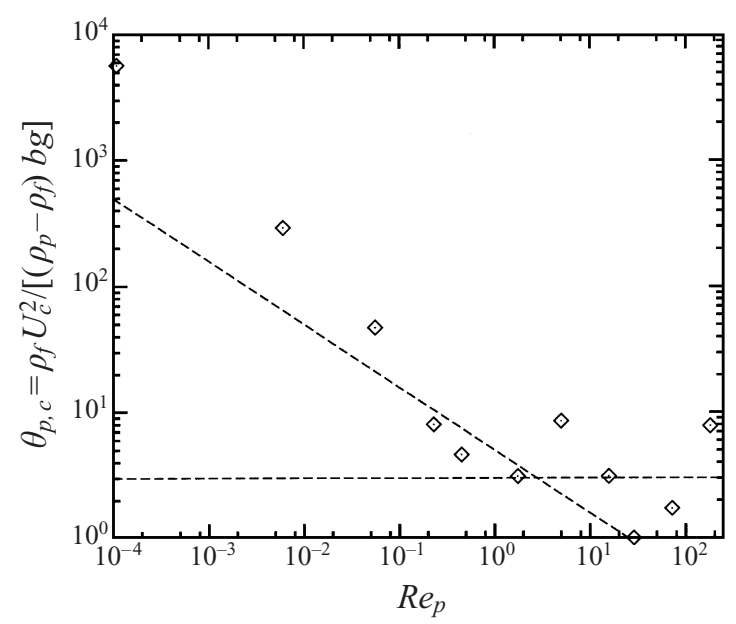

FIGURE 6. The variation of the critical particle Shields' parameter, $\theta_{p, c}$, with particle Reynolds number, $R e_{p}=b v_{T} / v$. The dashed lines represent the semi-empirical result (3.2).

provide a reasonable model since the separations $d, d_{1}$ and $d_{2}$ are typically much less than the vortex ring diameter, and hence the self-induced motion is relatively weak. This constraint also applies to the flow generated when a vortex ring strikes a wall obliquely, although the dynamics of a ring vortex are affected by the stretching of vortical elements and curvature. Lim (1989) studied experimentally the flow generated by a vortex ring impacting a wall, and observed that the section of vortex core upstream of the point of impact was further from the wall and initially travelled more slowly than the corresponding section downstream of the point of contact. These observations have some implications for the flow generated by a sphere impacting a wall obliquely. As already indicated, when $R e \leqslant O(1000)$ the component of the wake vortex downstream of the point of contact does not strike the wall because the secondary vortex plays a negligible role in the dynamics of the wake vortex. However, at higher Reynolds number the secondary vortex plays a more significant role in the dynamics of the wake vortex, and the wake vortex is observed to strike the wall.

\section{Resuspension of a layer of dust particles}

\subsection{Resuspension criterion}

The experiments described in the previous section gave insight into the flow generated by an impacting sphere. The conditions required for this flow to resuspend a thin layer of dust initially at rest on the wall, are now discussed.

The redistribution of a layer of dust, one or two particles thick, by a sphere impacting the wall at $\alpha=90^{\circ}$, was examined for a range of impact velocity $U$ and the dust particle properties (e.g. diameter $b$ and density $\rho_{p}$ ). For $U<10 \mathrm{~cm} \mathrm{~s}^{-1}$, the sphere was driven towards the wall by the motor and rod; higher impact velocities were obtained by releasing glass or steel spheres $20 \mathrm{~cm}$ above the wall, and allowing them to reach their terminal velocity prior to impact. The free-falling spheres, which were either steel ball bearings or glass beads with diameters $0.6 \mathrm{~cm}$ to $1.6 \mathrm{~cm}$, bounced on the wall, but the subsequent collisions did not appear to contribute significantly to the resuspension process. As the free-falling spheres descended they shed vortices which travelled more slowly than the spheres and were observed to interact with 
resuspended dust. The critical conditions for resuspension were determined by fixing the properties of the dust particles (table 1) and systematically increasing the speed of the impacting sphere, either by increasing the speed of the motor driving the Perspex sphere or by increasing the size or density of the spheres in the free-fall experiments, until particle motion was initiated in order to determine the critical impact velocity $U_{c}$. The experimental errors are associated with the incremental steps in the impact velocity to determine $U_{c}$. The ability of an impacting sphere to resuspend dust was characterized in terms of a 'particle' Shields' parameter, $\theta_{p}$, defined as

$$
\theta_{p}=\frac{\rho_{f} U^{2}}{\left(\rho_{p}-\rho_{f}\right) g b},
$$

and resuspension occurs when $\theta_{p} \geqslant \theta_{p, c}$, where $\theta_{p, c}$ is the critical Shields parameter. The threshold criterion for incipent sediment transport in channel flows is usually described by a Shields' parameter defined in terms of the ratio of bed shear stress, $\rho_{f} u_{*}^{2}$, and $\left(\rho_{p}-\rho_{f}\right) g b$ (Yalin 1977). The shear stress was not measured in our experiments; however the friction velocity scales with the impact velocity of the sphere and is the reason we have chosen to define a particle Shields' parameter (3.1). Figure 6 shows the variation of the critical particle Shields' parameter $\theta_{p, c}$ with the particle Reynolds number, $R e_{p}=v_{T} b / v$, based on the terminal fall velocity $v_{T}$ and diameter of the dust particles. When the dust particles are large $\left(R e_{p} \gtrsim 1\right)$, the critical Shields parameter was found to be approximately constant, but $\theta_{p, c}$ increased significantly as the dust particles became finer $\left(R e_{p} \ll 1\right)$.

The variation of $\theta_{p, c}$ with $R e_{p}$ may be understood by considering the conditions for incipient motion. The wake vortex passing over the layer of dust particles generates a boundary layer of thickness $a\left(v / U_{c} a\right)^{1 / 2}$ on the wall, and an inner viscous sublayer of thickness $O\left(v / U_{c}\right)$. When the dust particles are larger than the viscous sublayer, the velocity of the fluid over the dust is $O\left(U_{c}\right)$, and consequently the condition for the inertial lift force, $\rho_{f} \pi b^{2} U_{c}^{2} / 4$, to exceed the gravitational force $\pi b^{3}\left(\rho_{p}-\rho_{f}\right) g / 6$ reduces to $\theta_{p, c} \approx O(1)$. When the dust particle is smaller than the viscous sublayer, the velocity of the fluid over the particle is $O\left(U_{c}^{2} b / v\right)$ (Tennekes \& Lumley 1992) so that the inertial lift force due to the horizontal velocity in the viscous sublayer is $O\left(U_{c}^{4} b^{4} \rho_{f} / v^{2}\right)$ (see Krishnan \& Leighton 1995). The fall velocity of the dust particles characterized by $R e_{p} \ll 1$ is $v_{T}=g b^{2}\left(\rho_{p}-\rho_{f}\right) / 18 v \rho_{f}$, so that the condition that the inertial lift force exceeds the gravitational force reduces to $\theta_{p, c} \approx O\left(1 / R e_{p}^{1 / 2}\right)$. A semi-empirical condition for resuspension is

$$
\theta_{p, c} \approx \frac{5.0}{R e_{p}^{1 / 2}} \quad \text { for } R e_{p} \lesssim 1, \quad \theta_{p, c} \approx 3.0 \text { for } R e_{p} \gtrsim 1,
$$

where the multiplicative constants are determined from the experimental data. A comparison between the relationship (3.2) and the experimental results is given in figure 6. Whilst there is scatter in the experimental data, which we attribute to the difficulty of observing the exact conditions for the incipent motion, the general trend of the experimental results is captured by our prediction. We note, however, that the scatter is too great to confirm the exponent $n=1 / 2$ in the $\theta_{p, c} \approx R e_{p}^{-n}$ scaling for $R e_{p} \ll 1$. Other factors may be important in characterizing the critical conditions for resuspension when the dust particles are of a size comparable to the impacting sphere.

An examination of the resuspension of a thin layer of dust by an obliquely impacting sphere showed that the critical impact velocity was insensitive to $\alpha$ in the range $64^{\circ}$ to $90^{\circ}$. The insensitivity of $U_{c}$ to $\alpha$ may be anticipated on the grounds that 

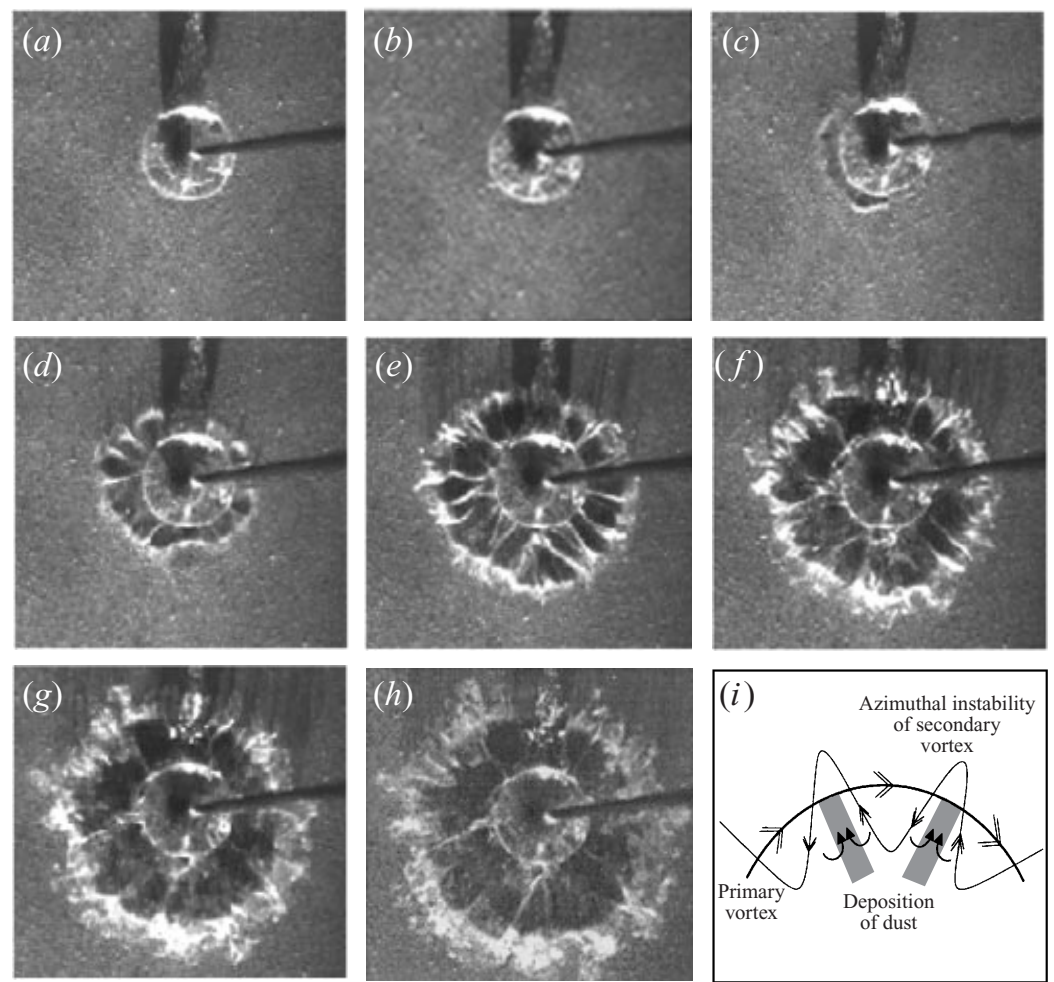

FIGURE 7. Images showing the redistribution of a layer of Pliolite $(100-150 \mu \mathrm{m}$ in diameter), by a sphere striking the wall at $\alpha=90^{\circ}$ with an impact velocity $U / U_{c}=3.3(R e=3100)$. Image $(a)$ corresponds to the time of impact, and the time difference between adjacent images is $a / U$. For comparison see figure 2, but note that the time difference between each image in figure 2 is $2 a / U$. (i) A schematic illustration of the mechanism by which the radial pattern is generated (the double arrows denote the direction of the vorticity).

the angle of impact sets a preferred direction for the wake vortex to leave the sphere, but does not significantly affect the speed of the fluid over the sediment, which is set by the speed of the impacting sphere. Consequently, the resuspension criterion (3.2) also applies to the case when the sphere strikes the wall obliquely, although the shape of the eroded region is dependent on $\alpha$.

\subsection{Resuspension of a thin layer of dust}

Figure 7 shows a series of images of the redistribution of a thin layer of Pliolite particles (diameter 100-150 $\mu \mathrm{m})$, due to the impact of sphere $\left(\operatorname{Re}=3100, \alpha=90^{\circ}\right.$, $U / U_{c}=3.3$ ). Following the sphere impact, the wake vortex detached, struck the wall and pushed (or rolled) dust particles out radially. The time difference between each image is $a / U$, and should be compared with the corresponding images of the wake vortex in figure 2. Figure 7 shows that the erosion pattern quickly developed alternate light and dark radial bands where the (white Pliolite) dust is respectively deposited and eroded. This pattern is generated because the secondary vortex develops an azimuthal instability as it is strained by the wake vortex: the mechanism of this instability has been studied in detail for vortex rings impinging walls (Orlandi \& Verzicco 1993). The azimuthal wavenumber of the secondary vortex was estimated by counting the number of alternate light and dark bands and was observed to increase with $R e$; for 


\begin{tabular}{llcccr}
\hline \multicolumn{1}{c}{ Material } & \multicolumn{1}{c}{$R e_{p}$} & $b(\mu \mathrm{m})$ & $\rho_{p}\left(\mathrm{~kg} \mathrm{~m}^{-3}\right)$ & $v_{T}\left(\mathrm{~cm} \mathrm{~s}^{-1}\right)$ & $U_{c}\left(\mathrm{~cm} \mathrm{~s}^{-1}\right)$ \\
Silicon carbide & $1.1 \times 10^{-4}$ & 4.5 & 3200 & 0.0025 & $100^{*}$ \\
Silicon carbide & $6.0 \times 10^{-3}$ & 17 & 3200 & 0.035 & $33.0^{*}$ \\
Pliolite & 0.056 & $100-150$ & 1030 & 0.038 & 2.11 \\
Silicon carbide & 0.23 & 54 & 3200 & 0.40 & 10.0 \\
Pliolite & 0.45 & $300-350$ & 1030 & 0.15 & 2.11 \\
Pliolite & 1.8 & $450-500$ & 1030 & 0.37 & 2.11 \\
Polystyrene & 5.0 & 330 & 1250 & 1.5 & 8.15 \\
Polystyrene & 16 & 950 & 1031 & 1.6 & 2.97 \\
Pliolite & 29 & $1200-1400$ & 1030 & 2.4 & 1.86 \\
Mustard seeds & 72 & 2000 & 1060 & 3.6 & 2.36 \\
Glass beads & 180 & 1200 & 2400 & 17 & $45.0^{*}$
\end{tabular}

TABLE 1. Properties of 'dust' particles used to determine the resuspension criterion; * denotes measurements using free-fall sphere experiments.

the particular experiment described in figure 7 , the azimuthal wavenumber is 10 , and is in the range calculated numerically by Orlandi \& Verzicco (1993). When a vortex ring, for instance a vortex shed from the sphere prior to the sphere striking the wall, collides with the wall, radial bands of Pliolite were not observed because the initial impact of a vortex ring pushed the dust to one side before the instability developed on the secondary vortex.

Following the impact of the sphere, dust is either pushed out radially and accumulates in a roughly circular ring, or is entrained (resuspended) by the wake vortex and propelled several sphere diameters away from the wall. The azimuthal instability of the secondary vortex does appear to play a role in the resuspension process by accumulating the dust into thick deposits which are then resuspended by the wake vortex. The timescale characterizing the resuspension process was set by the advective timescale associated with the flow past the sphere, $a / U$. Additional experiments were performed by illuminating the flow with a vertical light sheet passing through the centre of the sphere, in order to examine in more detail the resuspension process. These experiments indicated that the trapped secondary vortex (figure $2 a$ ) pushes Pliolite towards the sphere, resuspending a fraction and, following the sphere's removal, Pliolite was found to have been accumulated as a ring, near to the point of contact with the wall.

Figure $8(a)$ shows the variation of the diameter of the eroded region $D$ with the impact velocity, $U / U_{c}$, for $\alpha=90^{\circ}$. Following the impact of the sphere, the flow generated by the wake passing over the layer of the dust is maximum adjacent to the vortex and decreases in magnitude with distance from the centreline. As $U / U_{c}$ increases, the wake vortex is able to resuspend dust further from the centreline and consequently the diameter of the eroded region increases as shown in figure $9(a)$. The increase in the diameter of the eroded region was not brought about by the vortex ring becoming larger, but due to flow generated by the vortex having a more distant effect. When the Perspex sphere moved with a low impact velocity perpendicular to the wall $\left(U / U_{c} \approx 1\right)$, the region of dust particles disturbed by the wake vortex was typically not axisymmetric about the point of collision because the flow is not axisymmetric about the rod. The eroded region was measured from video film of the experiments and $D$ was taken to be the average of the width to the length of the eroded region. Included in figure $8(a)$ are results from free-fall experiments. The eroded region became more 

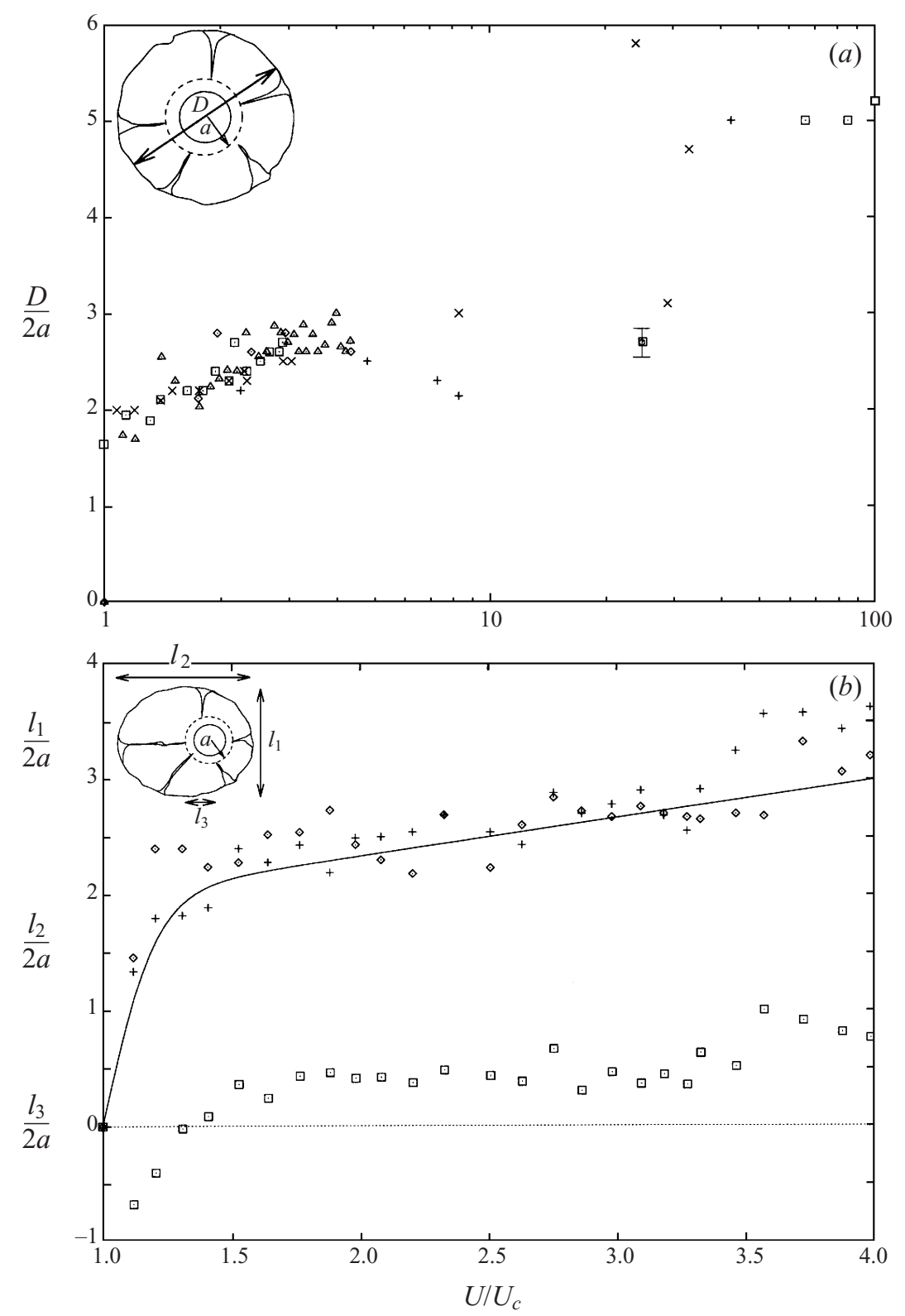

FiguRE 8. (a) The diameter of eroded region, $D$, generated by a sphere impacting at $\alpha=90^{\circ}$ as a function of $U / U_{c}$, where $U_{c}$ is the critical impact velocity, $U$ is the impact velocity of the sphere. The symbols $\square, \diamond,+, \times$ and $\triangle$ denote respectively when the layer consists of $450-500 \mu \mathrm{m}$ Pliolite particles, a fluorescine layer, $17 \mu \mathrm{m}$ silicon carbide, $0.95 \mathrm{~mm}$ polystyrene beads and $300-350 \mu \mathrm{m}$ Pliolite particles. For $U<10 \mathrm{~cm} \mathrm{~s}^{-1}$, a Perspex sphere was used, for $U>10 \mathrm{~cm} \mathrm{~s}^{-1}$ glass and steel balls of diameters 6-16 $\mathrm{mm}$ were used. (b) The geometry of the eroded region when a sphere collides at angle $\alpha=64^{\circ}\left(300-350 \mu \mathrm{m}\right.$ Pliolite), which is specified in terms of three length scales, $l_{1}(\diamond), l_{2}$ $(+)$ and $l_{3}(\square)$, indicated in the inset. The full curve shows the general trend of the data from $(a)$. 

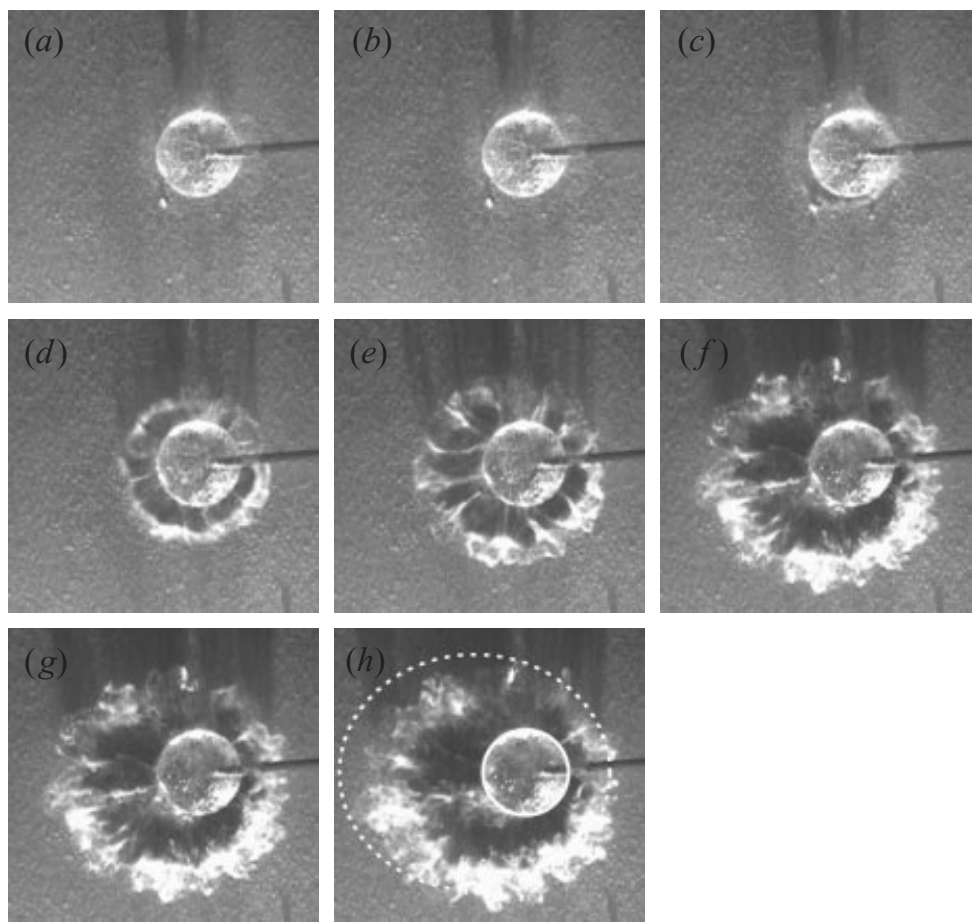

FIGURE 9. Images showing the redistribution of a layer of Pliolite $(100-150 \mu \mathrm{m}$ in diameter), by a sphere impacting at an oblique angle $\alpha=72^{\circ}$. The time difference between adjacent images is $a / U$. The sphere is moving from the right to the left, prior to colliding with the wall. The impact velocity is $U / U_{c}=3.3$, and corresponds to that given in figure 7. In $(h)$, the dashed elliptical curve shows the erosion pattern predicted by the simple geometrical model.

axisymmetric for higher impact velocities (even though the flow is not axisymmetric), and the diameter of the eroded region, $D$, was well-defined. Even though the dust properties were varied, the results in figure $9(a)$ collapsed onto a single curve, provided that the impact velocity is scaled by $U_{c}$. Additional experiments were performed to examine the resuspension of a thin mixed-layer of Pliolite and silicon carbide. In this case, two distinct regions of erosion were observed: one a large eroded area of pure Pliolite layer and the other corresponding to pure silicon carbide layer, suggesting that thin dust layers do not affect the dynamics of the wake.

When the sphere impacts the wall at an oblique angle the erosion pattern is not axisymmetric about the collision point. Figure 9 shows the resuspension of a layer of dust by a sphere impacting at an angle $\alpha=70^{\circ}$ and $U / U_{c}=2.0$, where the sphere is moving from the right to the left in the figure, and the camera is oriented to look slightly down on the wall. Here the eroded region is characterized by three lengthscales: breadth $l_{1}$, length, $l_{2}$, and distance of the centre of the eroded region from the collision point $l_{3}$ (as indicated in the inset on figure $8 b$ ). These lengthscales were measured as a function of $U / U_{c}$ for a number of impact angles; however we present results only for $\alpha=64^{\circ}$, which are plotted figure $8(b)$. For comparison, the diameter of the region corresponding to when the sphere struck the wall at $\alpha=90^{\circ}$ is indicated by the solid line. At low impact velocities $U / U_{c} \approx 1, l_{3}$ is negative, indicating that the eroded region does not extend around the sphere but is predominantly on one side giving a horseshoe shaped erosion pattern. As $U / U_{c}$ increases, the eroded region is extended around the sphere, initially becoming circular at $U / U_{c}=1.3$, 
but tending to an elliptical shape at higher impact velocities. The simple description of the flow generated by a two-dimensional dipolar vortex impacting a wall at $\alpha=64^{\circ}$, indicates that the dust is eroded by both sides of an impacting vortex when $U / U_{c}=\sqrt{1+\cos ^{2} \alpha}+\cos \alpha \sim 1.5$; the experimental observations indicate that resuspension occurs on both sides when $U / U_{c}=1.3$. The general trend for oblique impacts is that the erosion pattern tends to oblate (where $l_{2}>l_{1}$ ), and that the size of the erosion pattern is larger than when the sphere strikes the wall normally $\left(l_{1}, l_{2}>D\right)$.

A geometric model was developed to gain some understanding of the shape of the eroded region observed at high impact velocities. Observations of the flow around the sphere impacting the wall indicated that the wake and secondary vortex detached from the sphere at an angle $135^{\circ}$ to the rod, and appeared to propagate in a straight line, until close to the wall. For illustration, a section through such a cone is superimposed on to figure $4(\mathrm{~g})$, where the positions of the wake and secondary vortex are shown. This geometric model treats the trajectory of the vortex ring, composed of the wake and secondary vortex, as a circular cone subtended by an angle of $90^{\circ}$. The erosion pattern is interpreted as the bisection of a plane (the wall) and the circular cone, so that the predicted erosion pattern is elliptical, in general agreement with observations. For comparison, the erosion region predicted by the geometrical model is superimposed onto figure $9(h)$. Applying these ideas to the interpretation of figure $8(b)$, the geometrical model predicts that for $U / U_{c}=4.0$, the shape of the eroded region is characterized by $l_{1} / 2 a=3.8, l_{2} / 2 a=4.7, l_{3} / 2 a=1.2$, with an aspect ratio $l_{2} / l_{1}=1.20$. The geometrical model over-predicts the size of the elliptical eroded region because it neglects the dynamical effect of vortex stretching and vorticity production on the wall. Nevertheless, the predicted aspect ratio of the eroded region is in close agreement to the experimental measurement $l_{2} / l_{1}=1.20$ and the geometrical model does give some insight into the characteristics of the eroded region for high impact velocities.

\subsection{Resuspension of a thick layer of dust}

In the previous subsection, we examined the resuspension of a thin layer of dust, for which the volume concentration of resuspended dust was sufficiently low to have no significant effect on the dynamics of the flow. However, when the layer of dust is thick, the concentration of resuspended dust may be sufficiently high to generate a particle-driven gravity current, which transports dust from the site of resuspension.

We examined the dynamics of a particle-driven gravity current generated by a sphere impacting a thick layer of dust for $\alpha=90^{\circ}$, for which the gravity current tended to be axisymmetric about the point of collision. Figure 10 shows a time series of the flow generated by a steel sphere of radius $a=4 \mathrm{~mm}$, impacting a uniform layer of $17 \mu \mathrm{m}$ silicon carbide, whose surface loading $\sigma=0.6 \mathrm{~kg} \mathrm{~m}^{-2}$ corresponds to a layer of dust approximately $2 \mathrm{~mm}$ in depth. The layer of dust was illuminated by a light shone at a low grazing angle which allowed the surface relief to be visualized in detail. The sequence of images in figure 10 illustrates that initially dust is resuspended by the flow around the impacting sphere. The dust-laden flow collapses to produce a radial particle-driven gravity current, which transports dust a significant distance from the collision point. From the series of images we can see that the flow is initially turbulent; however the flow became laminar as the gravity current spreads out and viscous forces generated on the wall become important. At a later time, when the thickness of the gravity current is comparable to the size of the dust particles, the gravity current appears to stop, and the particles sediment to the dust layer. 

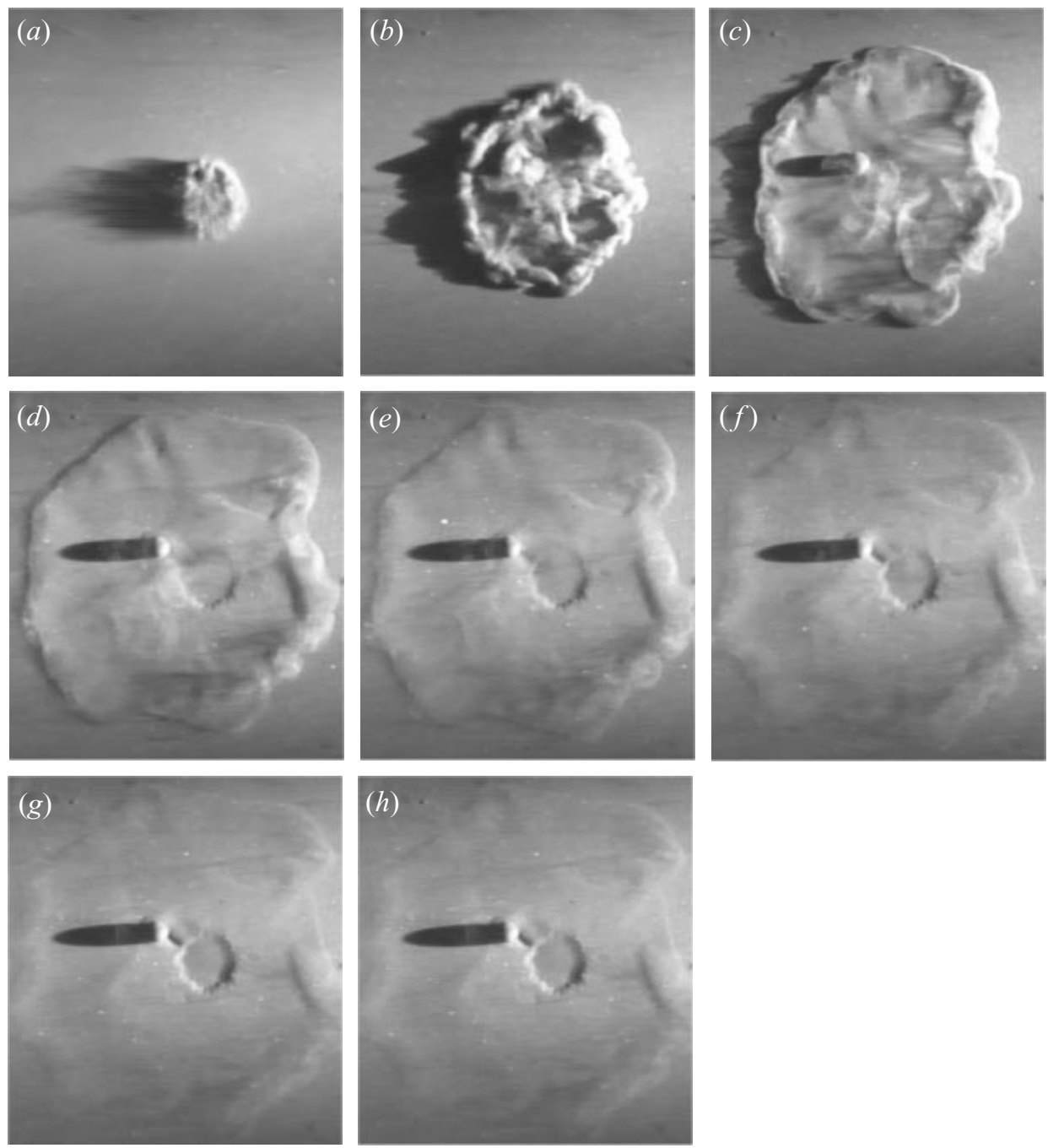

FIGURE 10. This series of images shows the resuspension of a thick layer of dust (silicon carbide, $17 \mu \mathrm{m}$ in diameter), by a steel sphere of diameter $4 \mathrm{~mm}$ released above the layer. The areal density of the silicon carbide is $0.6 \mathrm{~kg} \mathrm{~m}^{-2}$, which corresponds to a dust layer of $2 \mathrm{~mm}$. The first image corresponds to the time $0.1 \mathrm{~s}$ after the sphere struck the wall, and time difference between subsequent images is $0.5 \mathrm{~s}$. The sphere impacted with a velocity $U / U_{c}=4.8$, and bounced clear of the collision point.

The radial extent of the gravity current, $R(t)$, was measured as a function of the size and velocity of the impacting sphere, and of the properties of the dust particles. Figure 11(a) shows the variation of $R(t) / a$ with $v_{T} t / a$, when the size of the impacting steel sphere was fixed $(a=3 \mathrm{~mm})$, but the size of the silicon carbide dust particles was varied: $b=4.5,17$ and $54 \mu \mathrm{m}$. Figure 11(a) indicates that the time taken for the particle-driven gravity current to stop, $T_{M}$, after the sphere has impacted the ground is typically much less than $a / v_{T}$, which is the time taken for a dust particle to fall through the height to which the dust particles are initially resuspended. In addition, the maximum radial extent of the particle-driven gravity current, $R_{M}$, is much larger than the size of the eroded region, demonstrating the important role gravity currents play in transporting dust from the point of resuspension. 

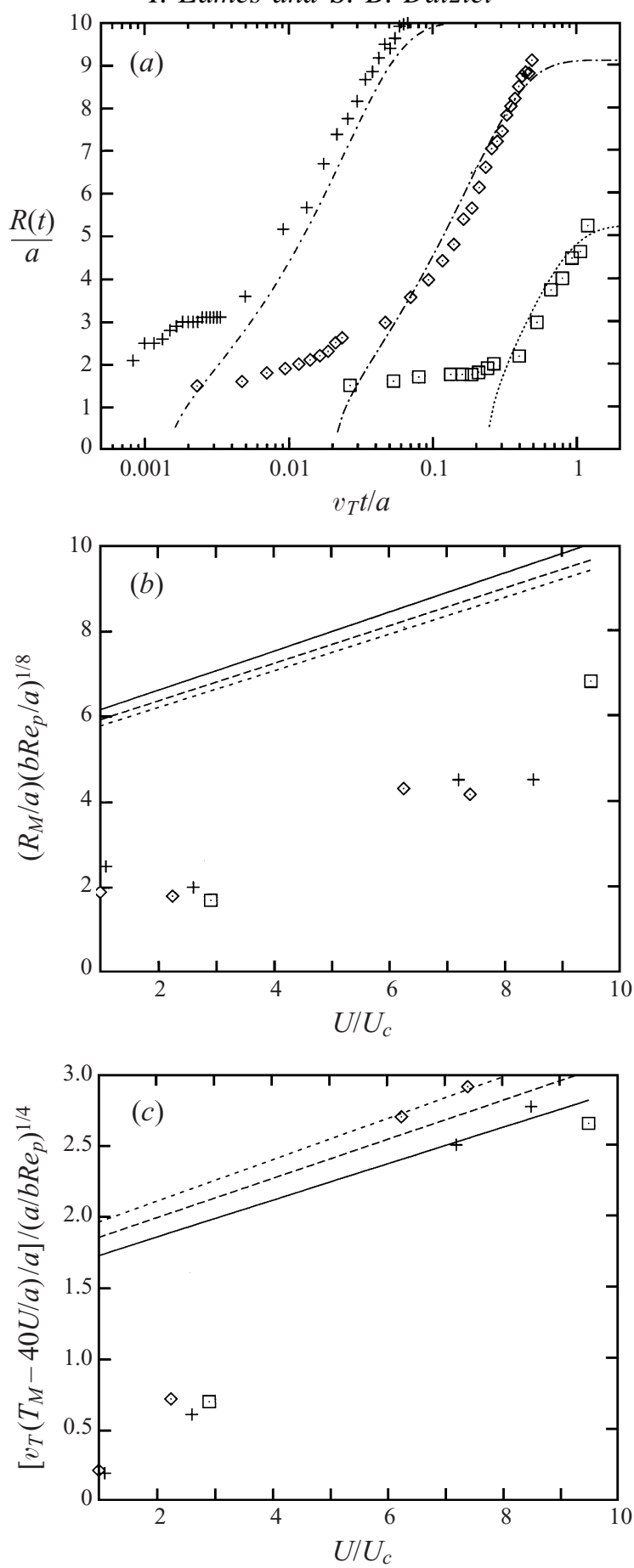

FiguRE 11. (a) The temporal variation of the radius of the gravity current, non-dimensionalized on the radius of the impacting sphere, for three particles sizes of (silicon carbide) dust: $\square, 54 \mu \mathrm{m}$ in diameter, $R e_{p}=0.23 ; \diamond, 17 \mu \mathrm{m}$ in diameter, $R e_{p}=6.0 \times 10^{-3} ;+, 4.5 \mu \mathrm{m}$ in diameter, $R e_{p}=1.1 \times 10^{-4}$. $(b, c)$ The variation of the maximum radial extent of the gravity current, $R_{M}$, and the time for the gravity current to stop, $T_{M}$, versus $U / U_{c}$. The size $a$ of the impacting sphere was varied: $\diamond, 3 \mathrm{~mm}$; ,$+ 4 \mathrm{~mm} ; \square, 5 \mathrm{~mm}$. Equation (3.4) is also plotted for these $a$-values:,$- 3 \mathrm{~mm} ;----, 4 \mathrm{~mm}$; .,$-- 5 \mathrm{~mm}$. 
The timescale associated with the hydrodynamic resuspension of the dust is set by the advective time-scale, $a / U$, which corresponds to $0.0088 \mathrm{~s}$ for the results shown in figure 11(a). The advective time-scale is less than the time between video fields, $0.02 \mathrm{~s}$, and consequently the details of the resuspension process could not be followed. However, a field-by-field analysis of the video tape permitted an examination of the growth of the dust cloud following resuspension which showed that for the three dust particle sizes considered, the size of the cloud of resuspended dust, $R(t) / a$, collapsed on to one curve when plotted against $U t / a$ for $U t / 2 a \leqslant 20.0$, where $t=0$ corresponds to time of impact. From figure 11(a), we see that for large time the development of the gravity current may be treated as though a turbulent patch of dust-laden fluid was released at time $40 a / U$ after the sphere has impacted the ground.

The dynamics of a particle-driven gravity current generated by the collapse of a volume of turbulent particle-laden fluid have been studied in detail experimentally and numerically by Dade \& Huppert (1995), Bonnecaze et al. (1995) and Bonnecaze, Huppert \& Lister (1996). Although the robust model of Bonnecaze et al. (1995, 1996) is strictly not applicable for the later development of the gravity current observed in these experiments when the flow is not turbulent, we still proceed to compare their predictions with our experimental measurements in order to gain insight into the transport following resuspension. Bonnecaze et al. (1995, see Appendix) calculated an analytical expression for the radial extent using a 'box model', which was first developed by Dade \& Huppert (1995) for two-dimensional particle-driven gravity currents. Bonnecaze et al. (1995) showed that the radial extend for a gravity current released at time $t=0$ is $R(t) / R_{M}=(\tanh (t / \tau))^{1 / 2}$, where $\tau$ is a timescale associated with the collapse of the gravity current and $R_{M}$ is the maximum radius. In fitting this expression to the experimental data, we estimate the timescale $\tau$ to be $\tau=2\left(T_{M}-40 a / U\right) / 3$, so that

$$
\frac{R(t)}{R_{M}}=\left(\tanh \left(\frac{t-40 a / U}{\frac{2}{3}\left(T_{M}-40 U / a\right)}\right)\right)^{1 / 2} .
$$

Expression (3.3) is plotted in figure 11(a), where $R_{M}$ and $T_{M}$ are experimental measurements. We should note that the radial extent of the gravity current, $R_{M}$, may be measured more accurately than the $T_{M}$ because of the difficulty in determining exactly when a thin gravity current has stopped.

Bonnecaze et al. $(1995,1996)$ parameterized the maximum radial extent, $R_{M}$, and characteristic collapse time of a particle-driven gravity current released from the origin at $t=0$ in terms of the initial volume of dust-laden fluid, $\mathscr{V}$, and the initial dust volume fraction, $\Phi$, as

$$
\begin{gathered}
R_{M}=1.9 \mathscr{V}^{1 / 3}\left(\frac{v_{T}}{\sqrt{g \Phi\left(\rho_{p} / \rho_{f}-1 \mathscr{V}^{1 / 3}\right.}}\right)^{-1 / 4}, \\
\tau=\left(\frac{4}{\pi F r^{2}}\right)^{1 / 4} \frac{\mathscr{V}^{1 / 3}}{v_{T}}\left(\frac{v_{T}}{\sqrt{g \Phi\left(\rho_{p} / \rho_{f}-1\right)^{1 / 3}}}\right)^{1 / 2} .
\end{gathered}
$$

Analysis of the silicon carbide layer after the impact showed that the dust layer thickness changed abruptly between the resuspended region and the undisturbed layer, indicating that dust within a distance $D / 2$ from the point of impact was resuspended, and not merely pushed to one side. In order to apply the scalings of Bonnecaze et al. (1995) an estimate of the initial volume $\mathscr{V}$ is required. We estimate 
the volume of dust-laden fluid to be $\mathscr{V}=\pi D^{3} / 8$, which is based on describing the initial volume of resuspended fluid as a circular cylinder of radius $D / 2$ and height $D / 2$. As a consequence of this assumption, the volume fraction of dust is estimated to be $\Phi=\left(\sigma D^{2} / 4\right) /\left(\rho_{f} D^{3} / 8\right)=2 \sigma /\left(D \rho_{f}\right)$, where $\sigma$ is the areal mass density of the dust. The maximum radial extent and collapse time vary according to

$$
\begin{gathered}
\frac{R_{M}}{a}\left(\frac{b R e_{p}}{a}\right)^{1 / 8}=1.9\left(36 \pi^{3}\right)^{1 / 2} \frac{D}{2 a}\left(\frac{\sigma}{2 a \rho_{f}}\right)^{1 / 8}, \\
\frac{\left(T_{M}-40 a / U\right) v_{T}}{a}\left(\frac{a}{b R e_{p}}\right)^{1 / 4}=\left(\frac{1}{9 F r^{2}}\right)^{1 / 4} \frac{D}{2 a}\left(\frac{\sigma}{2 a \rho_{f}}\right)^{-1 / 4},
\end{gathered}
$$

where the fall velocity $v_{T}$ is estimated using the Stokes linear drag law consistent with the approximation $R e_{p} \ll 1$. It is important to note that $R_{M}$ and $T_{M}$ are functions of the impact velocity through $D / 2 a$, whose variation with $U / U_{c}$ is shown in figure 8. Another important point is that the volume fraction of resuspended dust, $\Phi$, is dependent on the size of the impacting body because the mass of dust resuspended increases with $a$ as $O\left(\sigma a^{2}\right)$, the volume of dust-laden fluid $O\left(a^{3}\right)$ increases more rapidly, and so the mass fraction, $\Phi \sim O\left(\sigma /\left(\rho_{f} a\right)\right)$, decreases.

A series of experiments was undertaken using three sizes of impacting sphere and three grades of silicon carbide to test the scalings (3.4), and the variation of $R_{M}$ and $T_{M}$ with $U / U_{c}$ is plotted in a dimensionless form in figure 11(b,c). Expressions $(3.4 a, b)$ are plotted using data from figure $8(a)$ to determine a relationship between $D / 2 a$ and $U / U_{c}$ over the range $1<U / U_{c} \leqslant 10$. A comparison between measurements and the prediction (3.4) indicates that the gravity current propagates a shorter distance than can be accounted for by a turbulent particle-driven gravity current model. The robust model of Bonnecaze et al. is expected to describe the initial turbulent gravity current dynamics but the viscously dominated motion observed at later time, which effectively sets the run out distance, is not a feature of their model. While we anticipate the scalings (3.4) apply to gravity currents generated by large bodies resuspending dust, they appear to over-estimate the maximum radial extent, and the general trend of $R_{M}$ and $T_{M}$ with impact velocity is not predicted. The precise effect of viscous forces on particle-driven gravity current dynamics still requires study and these experimental results may go some way to validating future mathematical models.

\section{Flow around a sphere moving away from the wall}

In this series of experiments, the sphere was initially in contact with the wall and was set into motion impulsively at $t=0$ by switching on the motor, which drove the sphere with a constant velocity away from the wall. When a sphere is impulsively set into motion, the flow is inviscid outside the thin boundary layer on the surface of the sphere and wall. Since the fluid velocity close to the wall decreases with time, the inviscid flow has some bearing on whether dust, which is initially at rest on the wall, will be resuspended. The inviscid flow around the sphere may be calculated using the method of images (Lamb 1932). From this solution we may calculate the variation of tangential fluid velocity, $v_{x}$, on the wall with radial distance from the centreline for $\alpha=90^{\circ}$ : the time difference between each curve is $0.2 a / U$. The results for this calculation are shown in figure $12(a)$. Viscous forces reduce the flow close to the wall and consequently the lift force experience by the dust is less than that anticipated from the inviscid flow. Figure 12(a) indicates that the flow, and consequently the inertial lift force experienced by particles on the wall, decreases rapidly with time so that the 

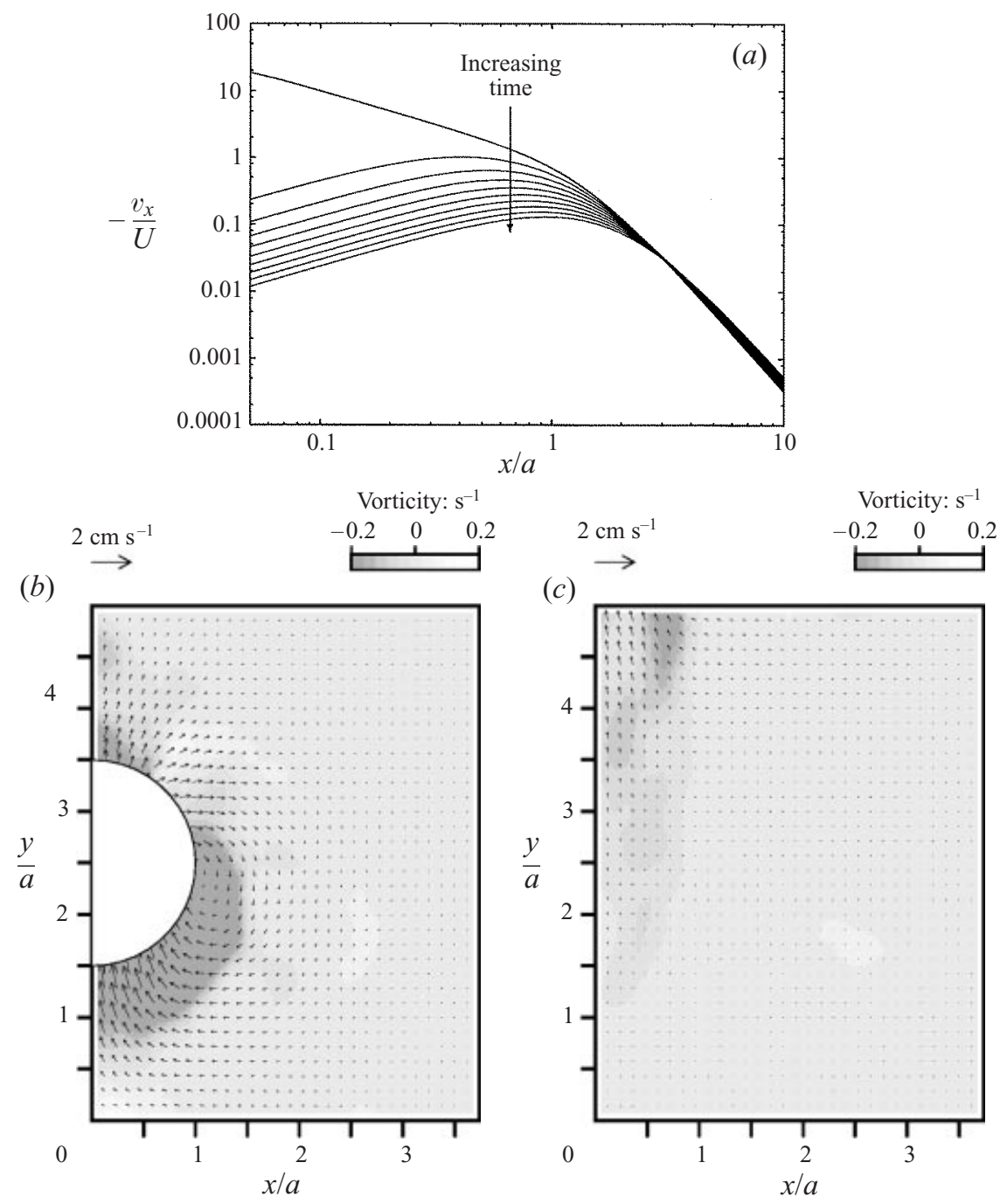

$(b)$

(c)

$\rightarrow$

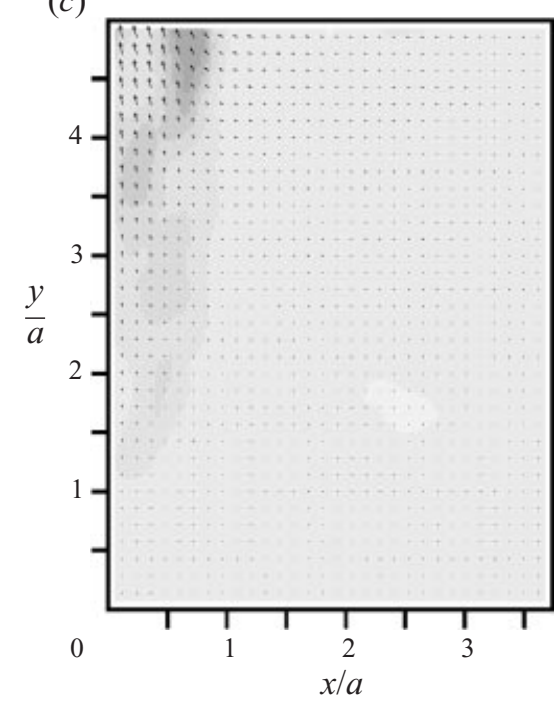

FIGURE 12. (a) The component of potential flow parallel to a wall generated by a sphere moving away from a wall is plotted as a function of radial position. The curves represent the flow at different times: the first curve corresponds to $U t / a=0.01$, and the time difference between the following curves is $0.2 a / U$. $(b, c)$ The velocity and vorticity field around a sphere moving away from a wall at times $U t / a=2.5,10$ after the sphere has been impulsively started from rest. The flow is characterized by a Reynolds number, based on the sphere velocity, of $R e=450$. The wake behind the sphere is characterized by the dark region, which denotes negative vorticity (pointing into the page). The flow near the wall persists for some time after the sphere has been removed, as indicated by $(c)$, and is consequently capable of driving a large volume of fluid away from the wall.

flow will have little effect on resuspension. In addition, the advective timescale $(a / U)$, is typically much shorter than the response time for the dust particles, and the dust will not respond to the flow generated by the sphere.

The velocity and vorticity field around the sphere are indicated in figure $12(b, c)$ for $R e=450, \alpha=90^{\circ}$, and correspond respectively to the times $U t / a=2.5$ and $U t / a=10.0$ after the motor was switched on. Figure 12(b) shows the wake behind the sphere after the sphere has translated a distance $2.5 a$. Vorticity generated on the 
surface of the sphere was either entrained by the wake vortex behind the sphere, causing it to grow with time, or deposited behind the sphere as trailing vorticity. For large time, the flow near the wall was dominated by the trailing vorticity, as indicated in figure 12(c), which shows the flow after the sphere has translated a distance 10.0a. The trailing vorticity advects a large (finite) volume of fluid, away from the wall, in the direction in which the sphere translated. For large time, the flow generated by the trailing vorticity decreased because of viscous dissipation, and was later disrupted by residual convection in the tank. The volume flux, $Q$, generated by the trailing vorticity may be estimated in an unbounded flow because it is related to the drag acting on the body, $F_{D}$, through $F_{D}=\rho_{f} Q U$ (Batchelor 1967). At high $R e$, the form drag is proportional to the cross-sectional area of the body and the dynamic pressure at the front stagnation point: $F_{D}=\frac{1}{2} \rho_{f} \pi a^{2} C_{D} U^{2}$, where the drag coefficient, $C_{D}$, varies from the value of 1.2 at $R e=100$ to 0.4 at $R e=10^{4}$. For the specific case of a sphere $\left(V=4 \pi a^{3} / 3\right)$, the volume flux is related to the drag coefficient through

$$
\frac{Q}{V U / a}=\frac{3}{8} C_{D},
$$

which indicates that the trailing vorticity advects $3 C_{D} / 8$ or $25 \%$ of a sphere's volume in the direction of motion. This estimation of the volume flux is only valid in unbounded flow where the flow is well-established. In the vicinity of the wall the volume flux is decreased because the flow is not well-established and the kinematic effect of the wall reduces the velocity normal to the wall, and consequently (4.1) remains an upper bound for $Q$.

The resuspension of dust was examined by introducing a thin layer of Pliolite particles $(100-150 \mu \mathrm{m}$ in diameter) on the wall. Following the removal of the sphere, one or two Pliolite particles which were initially in contact with the sphere, were observed to leave and remain trapped in its wake. In general, the Pliolite particles remained undisturbed because the flow adjacent to the wall was localized and decreased rapidly with time. These experiments were repeated using larger particles (mustard seeds, table 1), and we observed that slightly more particles were lifted from the wall. The general conclusion from these experiments was that a body moving away from a wall plays a negligible role in dust resuspension.

\section{Concluding remarks}

In this paper we examined experimentally the hydrodynamic mechanism of dust resuspension. The main results from this study are the following.

(a) The high-Re flow around a sphere moving towards and away from a rigid wall has been examined. The study has shown that when a sphere collides with a wall, the wake vortex which is initially behind the body, threads over the sphere's surface generating a secondary vortex. The coherent structure, composed of the wake vortex and secondary vortex, collides with the wall, pushing fluid which is adjacent to the wall to one side, which is subsequently entrained by the wake vortex. The secondary vortex was observed to develop an azimuthal instability, which was visualized by the disruption of a layer of Pliolite particles. When the sphere moved away from a wall, trailing vorticity generated on the surface of the sphere was observed to advect a large volume of fluid away from the wall.

(b) A criterion for resuspension by an impacting sphere was expressed in terms of a particle Shields' parameter, $\theta_{p}=\rho_{f} U^{2} /\left(\rho_{p}-\rho_{f}\right) b g$. When the dust-particle 
Reynolds number $\operatorname{Re}_{p}\left(=v_{T} b / v\right) \lesssim 1$, the dust is resuspended when $\theta_{p} \geqslant \theta_{p, c}$ where $\theta_{p, c} \approx 5.0 / R e_{p}^{1 / 2}$, and for $R e_{p} \gtrsim 1$ the critical Shields' parameter is $\theta_{p, c} \approx 3.0$. Above the critical Shields' parameter, the inertial lift force acting on the dust particles is sufficient to overcome the gravitational force and the dust particles are removed from the wall. The dust is entrained by the wake vortex and propelled several sphere diameters away from the wall. The geometry of the region eroded was determined as a function of impact velocity, angle of impact and the characteristics of the dust particles. The resuspension criterion indicates that the hydrodynamic mechanism of resuspension by saltating particles does play an important role in dust resuspension from soils.

(c) The dynamics of resuspensed dust were examined for the particular cases of high and low volume concentrations. When the sphere impacts a thick layer of dust, the mass fraction of resuspended dust is sufficiently large to generate a particle-driven gravity current which transports the dust a considerable distance from the impact site. The gravity current dynamics were studied and compared with the predictions of Bonnecaze et al. (1996) for turbulent particle-driven gravity currents. The gravity currents propagated a shorter distance than predicted by turbulent particle-driven gravity current models indicating the important role viscous forces play in the later development of these currents.

(d) A body moving away from a wall does not play a significant role in resuspending a layer of dust because the flow it induces on the wall decreases rapidly with time and distance from the sphere, and the advective timescale associated with the flow unsteadiness is much shorter than the response time of the dust particles. However, a body moving away from a wall transports a large volume of fluid away from the wall and consequently the flow around saltating particles leaving the ground play a significant role in increasing the vertical mass flux of dust.

Within a more general context, the results in this paper give insight into a wide range of multiphase flow problems where bubbles or rigid particles leave or approach walls or free surfaces. For instance, the sedimentation of large particles reduces the efficiency of sewage settling tanks, by resuspending fine particulate on the tank floor. The laying of oil pipes on the ocean floor or the dumping of containers will resuspend material on the sea floor, because the flow they generate is similar to that observed when a sphere impacts a wall. The flow pattern generated by a bubble (with surface contamination) rising to a free surface is similar to that observed when a sphere collides with a wall, and consequently it will play a significant role in enhancing interfacial gas transfer at the sea surface. A more detailed study of these flows is currently being undertaken.

I.E. acknowledges financial support from St. Catharine's College, through the Jeremy Howarth Fellowship. S. B. D. acknowledges the support of Yorkshire Water and the Isaac Newton Trust. Both authors gratefully acknowledge Dr Ian McEwan for providing a copy of Owen (1980) and Professor Dale Gillette for providing a copy of Owen (1986). We thank Dr Anne Rice for kindly granting access to her experimental results. The constructive criticisms from anonymous referees is acknowledged. 
Absil, F. G. J. \& Beugeling, G. L. H. 1984 The entrainment of small particles by a turbulent spot. In Atmospheric Dispersion of Heavy Gases and Small Particles - IUTAM Symp. Delft 1983, pp. 211-219. Springer.

Bagnold, R. A. 1941 The Physics of Blown Sand and Desert Dunes. Chapman \& Hall.

Batchelor, G. K. 1967 Introduction to Fluid Dynamics. Cambridge University Press.

Bonnecaze, R. T., Hallworth, M. A., Huppert, H. E. \& Lister, J. R. 1995 Axisymmetric particledriven gravity currents. J. Fluid Mech. 294, 93-121.

Bonnecaze, R. T., Huppert, H. E. \& Lister, J. R. 1996 Patterns of sedimentation from polydispersed turbidity currents. Proc. R. Soc. Lond. A 452, 2247-2261.

BRENNER, H. 1961 The slow motion of a sphere through a viscous fluid towards a plane surface. Chem. Engng Sci. 16, 242-251.

Clift, R., Grace, J. R. \& Weber, M. E. 1978 Bubbles, Drops and Particles. Academic.

DADE, W. B. \& HupPERT, H. E. 1995 A box model for non-entraining, suspension driven gravity surges on horizontal surfaces. Sedimentology 42, 453-471.

Gillette, D. A., Blifford, I. H. \& Fryrear, D. W. 1974 The influence of wind velocity on the size distribution of aerosols generated by the wind erosion of soils. J. Geophys. Res. 79, 4068-4075.

Greeley, R. \& Iverson, J. D. 1985 Wind as a Geological Process on Earth, Mars, Venus \& Titan. Cambridge University Press.

Krishnan, G. P. \& Leighton, D. T. 1995 Inertial lift on a moving sphere in contact with a plane wall in a shear flow. Physics Fluids 7, 2538-2545.

Lamb, H. 1932 Hydrodynamics. Cambridge University Press.

LiM, T. T. 1989 An experimental study of a vortex ring interacting with an inclined wall. Expts. Fluids 7, 453-463.

Lorenz, R. D. 1994 Huygens probe impact dynamics. ESA J. Eur. Space Agency 18, 93-117.

Magarvey, R. H. \& Maclarehy, C. S. 1968 Mass transfer and wake phenomena. AIChE J. 14, 260-265.

Orlandi, P. \& Verzicco, R. 1993 Vortex rings impinging on walls: axisymmetric and threedimensional simulations. J. Fluid Mech. 256, 615-646.

OwEN, P. R. 1980 The physics of sand movement. Unpublished note

OwEN, P. R. 1986 The erosion of dust by a turbulent wind. Unpublished note

Rice, M. A., Willetts, B. B. \& McEwan, I. K. 1996 Wind erosion of crusted solid sediments. Earth Surface Processes \& Landforms 21, 279-293.

Shao, Y., Raupach, M. R. \& Findlater, P. A. 1993 Effect of saltation bombardment on the entrainment of dust by the wind. J. Geophys. Res. 98, 12719-12726.

Swearingen, J. D., Crouch, J. D. \& Handler, R. A. 1995 Dynamics and stability of a vortex ring impacting a solid wall. J. Fluid Mech. 297, 1-28.

Tennekes, H. \& Lumley, J. L. 1992 A First Course in Turbulence. MIT Press.

Walker, J. D. A., Smith, C. R., Cerra, A. W. \& Doligalski, T. L. 1987 The impact of a vortex ring on a wall. J. Fluid Mech. 181, 99-140.

Wells, J. C. 1992 PhD Thesis, University of Grenoble.

Yalin, M. S. 1977 Mechanics of Sediment Transport. Pergamon. 